\title{
D2D-based Cooperative Positioning Paradigm for Future Wireless Systems: A Survey
}

\author{
Nadezhda Chukhno, Sergio Trilles, Joaquín Torres-Sospedra, Antonio lera, and Giuseppe Araniti
}

\begin{abstract}
Emerging communication network applications require a location accuracy of less than $1 \mathrm{~m}$ in more than $95 \%$ of the service area. For this purpose, 5G New Radio (NR) technology is designed to facilitate high-accuracy continuous localization. In $5 \mathrm{G}$ systems, the existence of high-density small cells and the possibility of the device-to-device (D2D) communication between mobile terminals paves the way for cooperative positioning applications. From the standardization perspective, D2D technology is already under consideration (5G NR Release 16) for ultra-dense networks enabling cooperative positioning and is expected to achieve the ubiquitous positioning of below one-meter accuracy, thereby fulfilling the 5G requirements. In this survey, the strengths and weaknesses of D2D as an enabling technology for cooperative cellular positioning are analyzed (including two D2D approaches to perform cooperative positioning); lessons learned and open issues are highlighted to serve as guidelines for future research.
\end{abstract}

Index Terms-Cellular networks, collaborative localization, cooperative localization, device-to-device communication

\section{INTRODUCTION}

A wide variety of emerging fifth-generation (5G) and beyond $5 \mathrm{G}(\mathrm{B} 5 \mathrm{G})$ applications demand high positioning accuracy, which, in turn, affects the performance of various location-based applications. The recent advances in the Global Navigation Satellite System (GNSS area, such as Precise Point Positioning (PPP) or Real-Time Kinematic (RTK), are enabling positioning accuracies below $10 \mathrm{~cm}$ outdoors. Despite positioning is more or less solved outdoors, the positioning accuracy still requires an improvement indoors. For instance, for indoor localization or positioning in dense urban settings, Wireless Local Area Network (WLAN) fingerprinting techniques can provide the accuracy of $3 \mathrm{~m}$ to $4 \mathrm{~m}$, while to

Manuscript received XX March 2021; revised XX XXXXXXX 2021; accepted $x X$ XXXXXXXXX 2021. Date of publication XX XXXXXXXXX 2021. The authors gratefully acknowledge funding from European Union's Horizon 2020 Research and Innovation programme under the Marie Skłodowska Curie grant agreement No. 813278 (A-WEAR: A network for dynamic wearable applications with privacy constraints, http://www . a-wear.eu//. Sergio Trilles has been funded by the postdoctoral Juan de la Cierva fellowship programme of the Spanish Ministry for Science and Innovation (IJC2018-035017-I). Joaquín Torres-Sospedra is funded by the Torres Quevedo Programme of the Spanish Ministry for Science and Innovation (PTQ2018-009981).

N. Chukhno and G. Araniti are with the University Mediterranea of Reggio Calabria, 89124 Reggio Calabria, Italy (e-mail: nadezda.chukhno@unirc.it; araniti@unirc.it). N. Chukhno and S. Trilles are with the Insitute of New Imaging Technologies, Universitat Jaume I, 12071 Castellón, Spain (e-mail: strilles@uji.es). J. Torres-Sospedra is with UBIK Geospatial Solutions, 12006 Castellón, Spain (e-mail: torres@ubikgs.com). A. lera is with the University of Calabria, 89124 Reggio Calabria, Italy (e-mail: antonio.iera@dimes.unical.it). A. lera and G. Araniti are also with the CNIT, Parma, Italy.

Corresponding author: N. Chukhno (nadezda.chukhno@unirc.it)

Digital Object Identifier: 10.1109/JSEN.202x.xxxxxxx achieve this result, one needs to maintain a large fingerprint database. Meanwhile, next-generation high accuracy positioning will require the accuracy of less than $1 \mathrm{~m}$ in more than $95 \%$ of the service area, including urban, outdoor, and indoor environments [1, 2]. It is already shown in [3, 4] that $5 \mathrm{G}$ New Radio (NR) technology can facilitate high-accuracy continuous tracking and positioning and confirmed that it is possible to achieve sub-meter localization accuracy over $99 \%$ of the time with the 3rd Generation Partnership Project (3GPP)-specified $5 \mathrm{G} / \mathrm{NR}$ parametrization.

Key prospects of $5 \mathrm{G}$ networks, such as increased bandwidth, small cells and high Mobile Terminal (MT) density, Deviceto-Device (D2D) communication capability, and multiple radio access technologies, are favorable for localization [5]. Specifically, $5 \mathrm{G}$ systems envisage scenarios, where MT may cooperate for achieving accurate positioning by directly exchanging necessary data through the D2D links. More technically, MT transmit their physical layer estimates, thereby accelerating local decisions. Then, higher layer information (e.g., position estimates between terminals or mobility information about the neighboring nodes) is exchanged between MT. This approach, for example, can be applied to use cases where the positioning accuracy is around a few meters to lessen the accumulative positioning error via cooperative positioning and, hence, improve the accuracy [6, 7].

Notably, in 5G delay-sensitive networks, a distributed D2D cooperative localization helps in reducing the delay to discover nearby nodes' locations, which is crucial, e.g., to perform specific intelligent control, such as self-driving [5]. A further feature paving the way for cooperative positioning applications in $5 \mathrm{G}$ networks is the availability of high density small cells, 
favouring D2D communication between MT. For example, in view of future Vehicle-to-Everything $(\mathrm{V} 2 \mathrm{X})$ scenarios, D2D communications are already considered in $5 \mathrm{G}$ NR Release 16 [8] as a valued means to enable ultra-dense cooperative localization. Due to this, D2D-aided cooperative positioning is expected to achieve the ubiquitous positioning of below onemeter accuracy [9], thereby fulfilling the $5 \mathrm{G}$ requirements [1]. Motivated by the aforementioned emerging interest in the subject, this paper focuses on integrated D2D positioning systems.

Differently from the previous studies on D2D and/or 5G technologies that fall into communication or multimedia sectors, this survey is devoted to positioning aspects. In this survey, we attempt to provide a holistic overview of the collaborative localization methods using D2D technology and the integration of the transmission and localization components of wireless systems. The survey investigates the state-of-the-art on D2D communication capabilities used for localization, thus acting as a glue between these two promising technologies for satisfying the ever-growing requirements of $5 \mathrm{G}$ and $\mathrm{B5G}$ networks. Summarizing, in this work, we concentrate on answering the following questions: (i) can $[2 D$ technology help positioning by increasing its accuracy?; (ii) can the cellular network provide effective support to positioning?; and (iii) what are pros and cons of D2D-based cooperative localization?

The survey's key take-away points are: (i) there are two ways to exploit D2D for cooperative localization. (ii) cooperative D2D positioning provides increased accuracy and extends coverage compared to non-cooperative methods. (iii) Cooperative $D 2 D$ positioning allows for relative position estimation, even in the absence of reference stations. (iv) $5 G$ mobile networks have enhanced positioning capabilities in comparison with previous generations. And (v) cooperative D2D-aided cellular positioning can facilitate sub-meter localization accuracy demanded by 5 G applications.

The remainder of this paper is organized as follows. We describe the application scenarios for collaborative positioning over 5G systems and used technologies in Section II Further, we provide a summary of positioning methods in Section [II] In Section IV, we review the state-of-the-art on $\mathrm{D} 2 \mathrm{D}$ technologies and provide a brief comparison of thereof, as well as discuss D2D-based cooperative localization systems. We summarize the lessons learned and open issues to be investigated in Sections $\mathrm{V}$ and $\mathrm{VI}$, respectively. The paper concludes with important general remarks collected in Section VII In Appendix II we collect the acronyms used throughout the paper.

\section{5G Positioning Applications and Enabling TECHNOLOGIES}

In this section, we discuss the application use cases for $5 \mathrm{G}$ localization and summarize their main requirements in Table [ Then, we review the technologies used for $5 \mathrm{G}$ localization.

As specified in [2, 10], 5G positioning services intend to support applications and verticals, such as Intelligent Transportation Systems (ITS), eHealth, Industry 4.0, with submeter positioning accuracy. Thus, many $5 \mathrm{G}$ verticals and applications pose ambitious system requirements for positioning accuracy (see Table I). For instance, in Location-based Services (LBS) and ITS scenarios, high accuracy is critical to new services and applications for both outdoors and indoors.

Regarding the Industry 4.0 use case, it is critical to locate assets and other moving objects (e.g., forklifts) on the factory floor. Similarly, in the logistic and transport industry, for example, in the use of drones, rail, and road transport, the need for object location determination exists. Further, in use cases with guided vehicles, Unmanned Aerial Vehicle (UAV), and objects involved in safety-related functions, also a high resilience in position availability is a key issue [10].

A further category, which demands high precision positioning, is mission-critical services. For instance, first responders may be located at all times during usual and critical operations, indoors as well as outdoors. Here, the level of positioning accuracy (and other Key Performance Indicators $\left(\right.$ KPI ${ }^{1}$ such as horizontal accuracy, vertical accuracy, positioning service availability, heading [11], latency for UE position estimation, corresponding positioning service level, UE speed, update rate, and Time to First Fix (TTFF) required is much more stringent than that demanded by local and regional regulatory requirements for commercial $5 \mathrm{G}$ users [10]. We summarize the main requirements for vertical use cases in Table I partial information of which is extracted from [8] (Section 6) and [10] (Section 9, Annex B).

Moreover, in particular applications and services, the network operator has to provide a customized localization service for various users requiring different performance levels. Hence, the support of several localization services is considered as a separate use case, which can be managed by relying on multiple technologies, for instance, $3 \mathrm{GPP}$ and non-3GPP technologies, as well as a combination of both $3 \mathrm{GPP}$ with non3GPP positioning technologies. It is important to mention that different localization methods are capable of offering different accuracy levels [12].

1) Non 3GPP Technologies: The exploitation of existing standards designed only for communications: currently, numerous wireless communication technology standards are available for WLAN, Wireless Sensor Network (WSN), and Internet of Things (IoT) applications. Examples can be Wireless Fidelity (Wi-Fi), ZigBee, Radio Frequency Identification (RFID), and Bluetooth Low Energy (BLE). These technologies do not provide any particular positioning capabilities. Instead, their transmitted signals are used and mixed to offer different levels of localization accuracy.

While RFID and BLE are typically used with proximity methods due to their limited transmission range, Wi-Fi technology has been successfully adopted in several positioning systems, usually leveraging fingerprinting techniques where accuracies at meter-level can be achieved in many conditions [12]. Wi-Fi and BLE have already been considered as complementary technologies in Long Term Evolution (LTE) Release 13 to enhance positioning in indoor environments [13], chiefly thanks to their wide diffusion.

\footnotetext{
${ }^{1}$ For example, TS 22.104 Clause 5.7 provides positioning requirements for horizontal and vertical accuracy, availability, heading, latency, and User Equipment UE speed in an industrial use case scenario.
} 
TABLE I

Vertical Use Cases for localization and Requirements [8, 10

\begin{tabular}{|c|c|c|c|c|c|}
\hline Vertical & $\begin{array}{l}\text { Regulatory and mission- } \\
\text { critical }\end{array}$ & Location-based & Industry & eHealth & Transport and logistics \\
\hline Use cases & $\begin{array}{l}\text { Public safety } \\
\text { First responder } \\
\text { Emergency call service }\end{array}$ & $\begin{array}{l}\text { Augmented reality } \\
\text { Wearables (tracking, } \\
\text { activity monitoring, and } \\
\text { emergency messages) } \\
\text { Advertisement push } \\
\text { Collaborative activities } \\
\text { (bike sharing, guidance } \\
\text { and flow management) }\end{array}$ & $\begin{array}{l}\text { Waste collection and } \\
\text { management } \\
\text { Connected enterprises } \\
\text { Smart retail }\end{array}$ & \begin{tabular}{|l} 
Connected healthcare \\
Patient tracking and \\
surveillance inside or \\
outside Hospitals \\
Location of emergency \\
equipment r outside \\
Hospitals (public spaces, \\
offices, etc.)
\end{tabular} & $\begin{array}{l}\text { Traffic monitoring, man- } \\
\text { agement, and control } \\
\text { Road-user charging (RUC) } \\
\text { Asset and freights tracking } \\
\text { Drone tracking } \\
\text { Supply chain visibility } \\
\text { Smart retail } \\
\text { Autonomous driving, } \mathrm{V} 2 \mathrm{X}\end{array}$ \\
\hline Environment of use & Both indoor and outdoor & $\begin{array}{l}\text { Both indoor and outdoor } \\
\text { in the } 5 \mathrm{G} \text { service area. }\end{array}$ & Outdoor & $\begin{array}{l}\text { Both indoor (Hospitals, } \\
\text { housing, offices, etc.) and } \\
\text { outdoor ( } 5 \mathrm{G} \text { service area) }\end{array}$ & Outdoor \\
\hline Accuracy & $\begin{array}{l}<1 \mathrm{~m} \text { horizontal, }<2 \mathrm{~m} \\
\text { vertical (indoor for floor } \\
\text { detection) and }<0.3 \mathrm{~m} \\
\text { vertical (relative) outdoor }\end{array}$ & $\begin{array}{l}2 \mathrm{~m} \text { horizontal, }<3 \mathrm{~m} \text { ver- } \\
\text { tical }\end{array}$ & $3 \mathrm{~m}$ horizontal & $\begin{array}{l}3 \mathrm{~m} \text { to } 10 \mathrm{~m} \text { horizontal, } \\
<3 \mathrm{~m} \text { vertical }\end{array}$ & $\begin{array}{l}0.1 \mathrm{~m} \text { to } 0.5 \mathrm{~m} \text { horizontal, } \\
0.1 \mathrm{~m} \text { to } 0.3 \mathrm{~m} \text { vertical }\end{array}$ \\
\hline Availability & $>95 \%(98 \%$ outdoor $)$ & $99 \%$ & $99 \%$ & from 90 to $99 \%$ & $99-99.9 \%$ \\
\hline Velocity & - & - & - & - & velocity $<3 \mathrm{~ms}^{-1}$ \\
\hline TTFF & $10 \mathrm{~ms}$ & $10 \mathrm{~s}$ & - & - & $10 \mathrm{~s}$ \\
\hline Latency & $1 \mathrm{~s}(5 \mathrm{~s}$ outdoor $)$ & $1 \mathrm{~s}$ & $60 \mathrm{~ms}$ & - & $150 \mathrm{~ms}$ \\
\hline Other KPI & $\begin{array}{l}\text { MCX Confidence Event- } \\
\text { triggered report }\end{array}$ & Normal mode & $\begin{array}{l}\begin{array}{l}\text { Very low energy } \\
\text { years })\end{array}\end{array}$ & - & $\begin{array}{l}\text { Low Energy, Antispoofing, } \\
\text { Antitampering }\end{array}$ \\
\hline
\end{tabular}

The ad-hoc solutions: Ultra-Wide Band (UWB) is known to be the most promising ad-hoc technology, which is able to achieve high-accuracy positioning in an indoor environment. This is thanks to the fact that a larger signal bandwidth ensures a higher time measurement resolution and, thereby, providing high-accuracy positioning estimations [12].

2) 3GPP Technologies: From a standardization perspective, positioning in 5G systems is discussed within the dedicated task in Release 16 [8]. Here, localization will be performed based on both (i) the NR uplink and downlink signals characteristics and (ii) the new network configurations and technologies such as sensors, Bluetooth, RFID, WLAN, GNSS, and Terrestrial Beacon Systems (TBS] [12]. Further, the main 5G technology enhancement consists in the employment and use of massive Multiple-Input-Multiple-Output (MIMO), beamforming, and Millimeter Wave (mmWave) directional transmissions. The mmWave frequency band exploitation yields a two-fold advantage, which is the large available bandwidth and the possibility to pack a large number of antenna elements even in small spaces (e.g., in a smartphone) [12]. Thanks to the wide bandwidth transmission, the former advantage makes it possible to enhance multipath robustness and time resolution performance of, e.g., Observed/Uplink Time Difference of Arrival (OTDOA/UTDOA localization approaches. Downlink OTDOA is assisted by UE, which means that UE receives downlink signals from the serving cell and multiple neighbors. Then, the UE determines the time difference between serving and neighbor cells to conclude on the position. Hence, it is a handset or UE based method, where UE is responsible for measuring time difference, and it requires a specific implementation at the UE side. Alternatively, UTDOA is a network-based location estimation method, and it does not require any $\mathrm{UE}$ interaction for position determination. Here, the uplink transmissions from UE are received by highly sensitive receivers, which will determine the time differences of arrival and, hence, UE position. The latter advantage facilitates single-anchor methods offering cm-level positioning accuracy, thereby addressing the bottleneck in indoor localization, which is a redundant ad-hoc infrastructure deployment.

From the application perspective, cellular technologies have been utilized for rough positioning for decades (e.g., 2G, 3G, 4G) for those cases where accuracy is less important. Such a positioning relies on the existing communication infrastructure and, hence, does not require dedicated deployments and significant maintenance costs. However, none of these previous cellular generations can meet future networks' positioning requirements (see Table $\mathrm{I}$. Conversely, $5 \mathrm{G} \mid \mathrm{NR}$ systems support several novel features, such as D2D communication and network densification, high carrier frequencies and large antenna arrays, large bandwidths, that make them favorable for the positioning of sub-meter accuracy. This means that $5 \mathrm{G}$ $\mathrm{NR}$ can provide positioning services with accuracy exceeding GNSS with no additional cost [14, 15].

\section{POSITIONING METHOdS}

There are multiple dimensions to be considered when developing a positioning system. In this section, we overview the existing localization method classifications. For example, Global Positioning System (GPS) can be included in distributed, absolute, and non-cooperative localization, whereas passive RFID tags coupled with RFID readers correspond to centralized, relative, and non-cooperative localization approaches.

\section{A. Indoor Localization vs. Outdoor Localization}

First and foremost, positioning can be divided into two types, depending on the positioning environment: outdoor and indoor. To this end, we also specify infrastructure-based and infrastructure-less indoor positioning systems as follows.

Indoor positioning is referred to as the last kilometer problem since the GNSS cannot work indoors [16]. Infrastructurebased Indoor Positioning System (IIPS) (is usually referred to 
as those systems that need the environment to be sensed to have indoor positioning (e.g., deploying BLE beacons, ultrasound receivers, etc.) Alternatively, Infrastructure-less Indoor Positioning is known as device-based positioning, wherein no additional infrastructure is required to operate, e.g., an indoor positioning system based on inertial measurements or magnetic field [17]. Outdoor positioning capabilities require regional or even global coverage compared to indoor environments, which are limited in size to rooms and buildings.

\section{B. Indirect Localization vs. Direct Localization}

Localization methods can be divided into two categories depending on the process of location estimation. It can be performed without relying on any intermediate parameters, i.e., directly from the received signal (direct) or relying on the first estimated intermediate parameters (indirect).

Multiple channel intermediate parameters' measurements of the multipath environment, e.g., Received Signal Strength RSSI, Angle Of Arrival (AOA, Time Of Arrival (TOA), Angle Of Departure AOD, phase, or combinations of them, are used for the positioning algorithms depending on the radio technology [18, 19]. These methods are referred to as the indirect localization (also known as two-step localization) where first the intermediate parameters are estimated, and then the user's position is obtained by using geometrical or triangulation/multilateration manipulations [20]. The measurement phase is affected by uncertainty due to environmental changes that influence positioning estimates differently depending on the chosen underlying technology [21]. Localization performance also varies depending on the specific algorithm used in the localization-update phase. An example of indirect localization is uplink TDOA (UTDOA) used in LTE systems. Here, first, sensors estimate TDOA (UTDOA) from all incoming signals. Second, they transmit such estimates to a central node that determines the sources' position by multilateration.

Multiple direct localization methods, where the user's location is determined directly from the received signal, have been developed to combat the uncertainty caused by the environmental changes [20, 22]. Here, contrary to the traditional two-step localization, the location of the source is estimated directly from the data, without estimating intermediate parameters, such as the $\mathrm{AOA}$ s of the Line-of-Sight (LOS) paths. Direct localization requires that the signals from multiple Base Stations $(\overline{\mathrm{BS}})$ ), or a function of them, have to be obtained by a fusion center to perform the user location estimation. For example, in [20], instead of performing triangulation with the strongest signals, all received multipath components are processed by a fusion center that determines the LoS directions, leading to an estimated position through triangulation.

\section{Active Localization vs. Passive Localization}

Localization systems could be divided into active and passive systems depending on whether the users have to carry the measurements on their devices such as smartphones, smartwatches, etc. More specifically, for active localization systems (also known as device-based active localization), users carry specific measuring devices [23]. It means that the entity being detected and tracked carries the tag or any device attached. Alternatively, passive localization (also known as device-free passive localization) aids the device to estimate its location in the environment. This method provides the capability of tracking and localizing entities not carrying any devices nor participating actively in the localization process. However, in real-life applications, the accuracy of passive localization is limited due to such effects as the environmental noises, multipath, among others [24].

\section{Centralized Localization vs. Distributed Localization vs. Decentralized}

Localization schemes can be classified depending on the place where the computation is performed [6]. According to this classification, distributed and decentralized localization is sometimes referred to as self-localization, whereas centralized localization is also known as remote localization. In centralized positioning, the central processor collects all location and measurement data from the anchors to calculate unlocalized nodes' positions jointly. Moreover, centralized approaches are supposed to offer a more accurate location performance since the information about the entire network is available in this case. Inversely, only local information exchange is required in distributed systems as the computation is spread over the entire network. The advantage of the distributed approach consists in scalability and robustness to node failures. In decentralized positioning, there are different devices connected to the network, and each device can perform positioning independent of the other. For example, scenarios such as a smartphone receiving signals from BSs, from which it may infer a position estimate about its actual position fall in the decentralized category.

The previous general classification leads to different computation paradigms for positioning that include Cloud Computing, Mobile Cloud Computing, Fog Computing, Mist/Things Computing, and Edge Computing. However, the computation paradigm is not usually fully described when introducing a new regular positioning system. The main focus is the algorithm description and assessment rather than its practical implementation, which can be server-based or on-device. In contrast, for cooperative/collaborative systems, the trends suggest that the decentralized approach is the most popular in the recent few years [17].

\section{E. Absolute Localization vs. Relative Localization}

Depending on the way the positioning systems provide location information, absolute and relative localization methods can be defined. Absolute localization refers to localization in a single predetermined coordinate system. The coordinate system is usually given by a geographic coordinate system, such as latitude, longitude, and altitude in GPS localization or implied in anchor locations [25]. Relative localization refers to the localization method where the coordinate system may vary from node to node, e.g., in the case of one's neighbors or the local environment. Relative location without a given coordinate system is also known as a relative map or a relative configuration [26]. An absolute or a relative position 
estimation can be further specialized into a vertical and a horizontal position. The former term refers to the position estimation on the vertical axis or altitude, whereas the letter means positioning in a horizontal plane or $2 \mathrm{D}$ reference [12].

\section{F. Non-cooperative Localization vs. Cooperative Localization}

The locations of uses can be determined either based on only the measurements between $\mathrm{MT}$ and $\overline{\mathrm{BS}}$; , i.e., without the internode measurements between MT or by using the interagent communication. In a non-cooperative approach, communication is established only between agents and anchors (no inter-agent links). Thus, every agent has to communicate with multiple anchors $(\overline{\mathrm{BS}})$ ), demanding either long-range anchor transmissions or a high density of anchors [27]. In cooperative localization, agents are no longer required to be under the coverage of multiple anchors as inter-agent communication (though D2D) removes the need for high anchor density and long-range anchor transmissions [21]. Here, long-range BS transmissions are replaced with multihop communications among the densely located MTs.

In cooperative localization, agents can obtain information from both anchors and other agents within the communication range (e.g., see Figure in Abstract). Moreover, D2D links have higher Signal-to-Noise Ratio (SNR and lower probability of the Non-Line-of-Sight (NLOS) path. Hence, cooperative localization increases the accuracy and extends the coverage compared to non-cooperative localization, which is the focus of this work.

\section{D2D-Based Cooperative Positioning}

In this section, we first provide a comparison of the technologies and standards used for D2D communication. We then review the current trends in D2D-based cooperative positioning for both indoor and outdoor environments.

\section{A. D2D Technologies Comparison}

D2D communications help in disseminating users' identification data, thereby facilitating direct interaction between mutually close devices, which require respective discovery and identification. Further, collective content creation and exchange enable users located in proximity to share and receive the desired content opportunistically. D2D-based interaction facilitates nearby users to participate in collaborative activities and communicate with each other's devices, thus emphasizing socialization and leisure, among many others LBS [28].

D2D technologies may be classified into two general categories: sharing licensed cellular spectrum or using dedicated resources. The former one tends to be limited by power and spectrum management perspectives and by usage cost. In contrast, the latter one suffers from uncontrolled interference and offers no Quality of Service (QoS) guarantees due to the random access behavior of, e.g., Institute of Electrical and Electronics Engineers (IEEE) 802.11 protocol stack. On the contrary, Wi-Fi outperforms cellular technologies in data rates and energy efficiency and is considered as the dominant solution for D2D connectivity, supporting wearable aggregation nodes [28].

Hardware costs and power consumption profiles in WLAN and Bluetooth specifications are more suitable for wearables. However, LTE and 5G road maps provide a gigabit-per-second experience, although some wearable devices may not be able to entirely derive the benefit of $\mathrm{LTE}$ and $5 \mathrm{G}$ due to potential cost and hardware complexity [29, 30].

A practical protocol for supporting $\mathrm{D} 2 \mathrm{D}$ communications in cellular networks by jointly using Wi-Fi Direct and LTE is proposed in [31]. Here, the communication between the cluster head and cluster clients is performed over Wi-Fi Direct. Proposed D2D architecture shows good performance in terms of delay and traffic load to be supported by D2D connectivity, and the scheduler minimizes LTE packet delays, which leaves room for relaxed $\mathrm{Wi}-\mathrm{Fi}$ operations at reasonable transmission rates.

Most recently, authors in [32] proved that the best option for cooperative data delivery in terms of energy consumption is to select a relay and perform D2D transmission over LTE More specifically, the power consumption analysis from both the infrastructure and user device perspectives conducted in [32] indicates that the minimization of active interfaces and sending the data with the best possible data rate is required to save the energy consumption. Since Wi-Fi is a good option only for high data rate transmissions, if there is no need for that, communicating devices should keep only the LTE interface active to save power. Similarly, in [33], authors advocate the use of LTE sidelink transmissions to address the missioncritical requirements and target new broadband public safety applications.

It is important to mention that, for example, for scenarios and applications such as emergency management (i.e., rescue and critical applications), multiple standards are required [34]. For instance, low-power Wireless Personal Area Network (WPAN) standards such as IEEE 802.15.6 are more suitable for on-body communication, they support a variety of realtime health monitoring and consumer electronics applications. Hovewer, these standards are not designed for bodyto-body communication. For that case, the use of low-power WPAN|IEEE 802.15.4 (Zigbee), Wi-Fil fourth-generation mobile telecommunications (4G) LTE D2D is required, which can effectively extend the network connectivity and coverage. Further, for off-body communication, one of the enddevices should communicate through cellular networks or infrastructure-based networks (e.g., 4GILTE). To summarize, even if existing devices can already support multiple standards, existing protocol stacks are not smart enough to provide connectivity or routing between different network technologies, which poses one of the critical challenges for future wireless networks [34].

$5 \mathrm{G}$ wireless communication systems utilizes $\mathrm{NR}$ sidelink for D2D communication [36]. The central scenario of Release $16 \mathrm{NR}$ sidelink transmissions targets $\mathrm{V} 2 \mathrm{X}$, and $\mathrm{NR}$ services are no longer limited to the Cooperative Awareness Message (CAM) and the Decentralized Environmental Notification Message (DENM) compared to LTE. In addition, by using 
TABLE II

D2DTECHNOLOGIES COMPARISON

\begin{tabular}{|c|c|c|c|c|c|c|}
\hline \multicolumn{3}{|c|}{ Work } & Technology & Advantages / Features & Device Type & Application \\
\hline 28 & 31 & 32] & Wi-Fi & $\begin{array}{l}\text { Wi-Fi provides higher data rates and energy efficiency (due to lower transmis- } \\
\text { sion delay) compared to cellular technologies. The devices are recommended } \\
\text { to reduce the number of active radio interfaces and transmit with the best } \\
\text { possible rates to minimize total power consumption. Thus, Wi-Fil is used only } \\
\text { for high data rate services and is suitable for body-to-body communications }\end{array}$ & Wearables & $\begin{array}{l}\text { Collective activities, social- } \\
\text { ization and leisure, various } \\
\text { LBS }\end{array}$ \\
\hline$[\overline{32}$ & 33 & 34] & LTE & $\begin{array}{l}\text { If high data rates are not required, devices should keep only the LTElinterface } \\
\text { active to save power. 4G,LTE D2D are required for wearable off-body and } \\
\text { body-to-body communication to extend the network connectivity }\end{array}$ & IoT, wearables & Vary \\
\hline & [34] & & IEEE 802.15 .6 & $\begin{array}{l}\text { Low-power WPAN standards such as IEEE } 802.15 .6 \text { is more suitable for } \\
\text { on-body communication }\end{array}$ & Wearables & $\begin{array}{l}\text { Public safety and critical } \\
\text { applications }\end{array}$ \\
\hline & [34] & & IEEE 802.15 .4 & $\begin{array}{l}\text { For body-to-body communication, using IEEE } 802.15 .4[\mathrm{D} 2 \mathrm{D}] \text { is required, } \\
\text { which can extend the network connectivity in an effective manner }\end{array}$ & Wearables & $\begin{array}{l}\text { Public safety and critical } \\
\text { applications }\end{array}$ \\
\hline & 29 & & Bluetooth & $\begin{array}{l}\text { Bluetooth (and Wi-Fi) technologies are more suitable for wearables in terms } \\
\text { of hardware cost and power consumption. Depending on the wearable device } \\
\text { type, devices may not be able to take full advantage of [TE and } 5 \mathrm{GG} \text { due to } \\
\text { the cost and hardware complexity }\end{array}$ & Wearables & Vary \\
\hline & 35] & & \begin{tabular}{|l|l|}
$5 \mathrm{G}$ & $\mathrm{NR}$ \\
\end{tabular} & $\begin{array}{l}\text { NR sidelink ensures low-latency, high-reliability, and high-throughput trans- } \\
\text { missions, as well as a high connection density services }\end{array}$ & Mostly vehicles & $\begin{array}{l}\text { Advanced and remote driv- } \\
\text { ing, platooning, sensors }\end{array}$ \\
\hline
\end{tabular}

a wider bandwidth, flexible massive antenna systems, and beamforming, $\mathrm{NR}$ V2X will provide more precise timing and accurate measurement of equivalent signal techniques in LTE$\mathrm{V} 2 \mathrm{X}$ [37]. The new NR sidelink use cases require low-latency, high-reliability, and high-throughput transmissions, as well as a high connection density. To this end, four new designs are introduced to $\mathrm{NR}$ sidelink: (i) in addition to broadcast, also unicast and multicast are supported; (ii) the performance in terms of latency is improved by grant-free transmissions adopted in NR Uplink (UL) transmissions; (iii) it improves the channel sensing and resource allocation procedures to mitigate collisions among different sidelink transmissions initiated by various MT;; (iv) high connection density is achieved by supporting congestion control and QoS management. For more detail, interested readers are referred to [35]. We summarize our main findings on D2D technology comparison in Table II

\section{B. Review of D2D-based Cooperative Positioning}

As discussed above, 5G supports D2D communication via sidelink which can be controlled by the BS. The UL, Downlink (DL), and sidelink communications can operate over two frequency bands: sub-6 GHz and above $24 \mathrm{GHz}$ (known as mmWave band). Whereas much effort has been done in the sub-6 GHz band [38], mmWave frequency spectrum is of special interest for localization [14, 39] thanks to the large available bandwidth, sparse channels and large antenna arrays. In [40], authors investigate a real-time positioning based on mmWave D2D links, which will ensure reliable communication in 5G cellular networks. Authors state that mmWave signals can provide up to centimeter level accuracy. Similarly, in [41], authors examine vehicle positioning using 5GD2D mmWave signals. Obviously, what clearly emerges is that if all MTs' positions are unknown, the absolute position will not be obtained, which means that $D 2 D$ itself can provide only relative measurements. Hence, the main interest shifts towards integrated positioning systems.

1) Indoor Positioning: We first focus on the indoor environment. Recall that cooperative positioning is of extreme importance for indoor scenarios where GPS signals are usually weak to provide sufficient position information. In [42], a cooperative localization scheme for WLAN fingerprinting is proposed to improve the accuracy of fingerprint-based location estimations affected by the random environmental changes. More precisely, instead of regenerating the radio map (typical for fingerprinting), users cooperate by exchanging both their real-time RSSI measurements and their location estimations and processing them with the aid of the Self-Organizing Map structure. Allowing collaboration among users increases the accuracy of the non-cooperative fingerprinting approach, and the performance enhancement is higher for denser user populations. In [43], authors exploit cooperative positioning among multiple pedestrians to reduce the accumulative error of dead reckoning carried out only by means of the smartphone low-cost sensors (infrastructure-less system) and Wi-Fi interface by exchanging RSSI measurements. Here, the participants communicate and measure the range $\mathrm{Wi}-\mathrm{Fi}$ ranging) between each other and then correct each of their positions to make them consistent with the range information. The results demonstrate an accuracy comparable to GPS when a high number of pedestrians cooperate in collaborative positioning. The proposed cooperative scheme solves the typical problem of the indoor positioning system, which is the cost (as positioning is performed on a device) and accuracy (improved by means of D2D technology). Similarly, in [21], cooperation between nodes in UWB system results in high accuracy and robustness.

A collaborative method based on D2D communication to enhance the indoor positioning accuracy using only direct communication to nearby devices and fingerprinting is introduced in [7]. The main idea behind this approach consists in verifying the fingerprinting positioning by TOA-based distance passed through the D2D link to rectify the erroneous response of BS Communication with other devices is repeated several times within a short period to increase the confidence level in the verification process. However, power consumption restrictions have not been considered, while there is a growing 
TABLE III

The State-OF-THe-ART on D2D Based Positioning

\begin{tabular}{|c|c|c|c|c|c|}
\hline Work & Env. & Methods & Approach & D2D approach & Accuracy \\
\hline$|\overline{6}|$ & Indoor & $\begin{array}{l}\text { IoT and [5G localization and } \\
\text { D2D }\end{array}$ & $\begin{array}{l}\text { In }[\mathrm{IoT} \text { and }[5 \mathrm{G} \text { localization, the value of inter-device measurements } \\
\text { helps to surpass current accuracy and coverage levels by at least an } \\
\text { order of magnitude }\end{array}$ & $\begin{array}{l}\text { Pseudo-range } \\
\text { estimates }\end{array}$ & $\begin{array}{l}\text { sub-meter } \\
\text { accuracy }\end{array}$ \\
\hline 7 & Indoor & RF Fingerprinting and D2D & $\begin{array}{l}\text { The main idea is to verify the fingerprint positioning response by } \\
\text { TOA based distance transmitted over D2D communication to nearby } \\
\text { devices in order to rectify the erroneous response of BS }\end{array}$ & $\begin{array}{l}\text { Pseudo-range } \\
\text { estimates }\end{array}$ & $\begin{array}{l}5.34 \mathrm{~m} \quad \text { (static) } \\
5.59 \mathrm{~m} \text { (dynamic) }\end{array}$ \\
\hline $\mid 21]$ & Indoor & UWB and D2D & $\begin{array}{l}\text { The cooperation between nodes is used to increase the accuracy and } \\
\text { robustness of UWB systems }\end{array}$ & $\begin{array}{l}\text { Pseudo-range } \\
\text { estimates }\end{array}$ & $\begin{array}{l}\text { accuracy improve- } \\
\text { ment compared to } \\
\text { non-cooperative }\end{array}$ \\
\hline$\overline{42}$ & Indoor & \begin{tabular}{|l|} 
WLAN Fingerprinting and \\
D2D
\end{tabular} & $\begin{array}{l}\text { Iterative update of the initial estimated location information from } \\
\text { WLAN fingerprinting by exchanging both real-time RSSI measure- } \\
\text { ments and location estimations between MT }\end{array}$ & $\begin{array}{l}\text { Location } \\
\text { information } \\
\text { exchange }\end{array}$ & $\begin{array}{l}\text { accuracy improve- } \\
\text { ment compared to } \\
\text { non-cooperative }\end{array}$ \\
\hline$|\overline{43}|$ & Indoor & $\begin{array}{l}\text { Dead reckoning and } \mathrm{D} 2 \mathrm{D} \\
\text { Wi-Fi ranging exchange) }\end{array}$ & $\begin{array}{l}\text { Cooperative positioning information exchange among multiple } \\
\text { pedestrians to reduce the accumulative error of dead reckoning } \\
\text { carried out with only the low-cost sensors and Wi-Filin smartphones }\end{array}$ & $\begin{array}{l}\text { Pseudo-range } \\
\text { estimates }\end{array}$ & less than $5 \mathrm{~m}$ \\
\hline$[44$ & Indoor & \begin{tabular}{|l|l|} 
Wi-Fi & Fingerprinting and \\
D2D & \\
\end{tabular} & $\begin{array}{l}\text { Collaborative } \mathrm{D} 2 \mathrm{D} \text { based method is used to eliminate redundant } \\
\text { fingerprints from a crowd-sourced database }\end{array}$ & $\begin{array}{l}\text { Location } \\
\text { information } \\
\text { exchange }\end{array}$ & $\begin{array}{l}\text { less than } 25 \mathrm{~m} \text { and } \\
50 \mathrm{~m}\end{array}$ \\
\hline (45) & Indoor & \begin{tabular}{|l|} 
RSSI of Wi-Fi and D2D \\
over Bluetooth
\end{tabular} & $\begin{array}{l}\text { RSSI of Wi-Fi contains information about users' relative distances, } \\
\text { and Bluetooth signals are exchanged between them to improve the } \\
\text { performance of existing indoor positioning methods }\end{array}$ & $\begin{array}{l}\text { Pseudo-range } \\
\text { estimates }\end{array}$ & $\begin{array}{l}1.34 \mathrm{~m} \\
\begin{array}{l}\text { (average), } 6.68 \mathrm{~m} \\
\text { (maximum) }\end{array}\end{array}$ \\
\hline 467 & Indoor & LTE (OTDOA) and D2D & OTDOA positioning is enhanced with $[\mathrm{D} 2 \mathrm{D}]$ cooperative techniques & $\begin{array}{l}\text { Pseudo-range } \\
\text { estimates }\end{array}$ & $\begin{array}{l}\text { accuracy improve- } \\
\text { ment compared to } \\
\text { non-cooperative }\end{array}$ \\
\hline $\mid \overline{5}$ & Outdoor & $5 \mathrm{G}$ and D2D & $\begin{array}{l}\text { The inter MT communications provide relative location information } \\
\text { between the MT that serve as a supplement to the BS MT links }\end{array}$ & $\begin{array}{l}\text { Pseudo-range } \\
\text { estimates }\end{array}$ & $\begin{array}{l}1 \mathrm{~m} \text { and below } \\
\text { (suggested) }\end{array}$ \\
\hline [39] & Outdoor & 5G and D2D & $\begin{array}{l}\text { The exchange of data between } \mathrm{MT} \text { and the network or between the } \\
\text { MT in } 5 \mathrm{G} \text { to increase the accuracy of, e.g., Time Difference Of } \\
\text { Arrival (TDOA-based method }\end{array}$ & $\begin{array}{l}\text { Pseudo-range } \\
\text { estimates }\end{array}$ & $\begin{array}{l}\text { sub-meter } \\
\text { accuracy }\end{array}$ \\
\hline$[\overline{14} \mid \overline{48}$ & Outdoor & \begin{tabular}{|l|l|}
$5 \mathrm{G}$ mmave and $\mathrm{D} 2 \mathrm{D}$ \\
\end{tabular} & $\begin{array}{l}\text { D2D is used for disseminating and computing location information } \\
\text { between vehicles }\end{array}$ & $\begin{array}{l}\text { Pseudo-range } \\
\text { estimates }\end{array}$ & $\begin{array}{l}\text { accuracy improve- } \\
\text { ment compared to } \\
\text { non-cooperative }\end{array}$ \\
\hline$[49$ & Outdoor & $\begin{array}{l}\text { Radio Frequency } \mathrm{RF} \text { fin- } \\
\text { gerprinting and } \mathrm{D} 2 \mathrm{D}\end{array}$ & $\begin{array}{l}\text { MT communicates not only with } \mathrm{BS} \text { to capture a series of RF } \\
\text { measurements but also with other } \mathrm{MT} \text { by leveraging the D2D } \\
\text { communication protocol }\end{array}$ & $\begin{array}{l}\text { Pseudo-range } \\
\text { estimates }\end{array}$ & $\begin{array}{l}\text { accuracy improve- } \\
\text { ment compared to } \\
\text { non-cooperative }\end{array}$ \\
\hline $\mid \overline{50}]$ & Outdoor & GNSS and 5G D2D & $\begin{array}{l}\text { Any-time and any-where seamless positioning by using the inte- } \\
\text { grated methodology of GNSS and D2D measurements }\end{array}$ & $\begin{array}{l}\text { Pseudo-range } \\
\text { estimates }\end{array}$ & $\begin{array}{l}56.2 \% \text { better } \\
\text { than for non- } \\
\text { cooperative }\end{array}$ \\
\hline $\mid 51$ & Outdoor & $\begin{array}{l}\text { Multi-Kalman Filter (MKF) } \\
\text { approach and D2D }\end{array}$ & $\begin{array}{l}\text { The combination of the interacting multiple model estimations } \\
\text { with the Multi-Kalman Filter (MKF) approach based on GPS and } \\
\text { Geographic Information System [GIS) big data }\end{array}$ & $\begin{array}{l}\text { Pseudo-range } \\
\text { estimates }\end{array}$ & \\
\hline
\end{tabular}

interest in developing more energy-efficient algorithms and protocols to support Green Communications, thereby reducing the environmental and economic impact. Likewise, the benefits of direct interconnection between nodes in terms of the localization accuracy (sub-meter) and coverage improvement for 5G IoT applications are demonstrated in [6].

In [44], a collaborative D2D-based method is used to remove the redundancy from the crowd-sourced Wi-Fi fingerprint database. Moreover, D2D is also used to provide privacy to the users by breaking the links between them and the data. Similarly to [43], authors deploy a localization system on devices rather than on infrastructure, thereby solving the problem of the infrastructure cost. Yet, as we see from Table III the accuracy of Wi-Fi fingerprints localization still has room for improvement. In [45], a framework for improving the performance of existing indoor positioning methods for smartphones with the help of information exchanging between users is designed. More precisely, the RSSI of Wi-Fi contains information about users' relative distances, and Bluetooth signals are exchanged among them for the purpose of assessing the probability distribution functions of users' states. The average error of the position estimates of the proposed system is $1.3357 \mathrm{~m}$, which can be considered as good performance for indoor localization performed of the device. The accuracy of the cooperative D2D-based localization scheme in [45] significantly outperforms the solutions proposed in the literature for low-cost indoor infrastructure-less positioning [43, 44].

As discussed in [46, 47], LTE cooperative localization technique, wherein the MT communicates with both eNodeB $\$^{2}$ (OTDOA positioning method supported by 3GPP) and other MT in proximity, can significantly improve the localizability in the network and enhance the accuracy which is highly beneficial to some applications, e.g., E911. It is demonstrated that cooperative localization is undoubtedly able to overcome problems of non-cooperative positioning (e.g., bad geometry, etc.) and, by increasing the number of collaborators, significantly improve the localization accuracy. Note that the synchronization problem between MTs that may appear in

${ }^{2}$ E-UTRAN Node B, also known as Evolved Node B (abbreviated as eNodeB or eNB), is an LTE BS. 
distributed cooperative localization systems and cause high accuracy error rate can be handled by inband D2D (that is, LTE sidelink) communication through the primary sidelink synchronization signal (for those MT that are under the eNodeBs coverage). However, synchronization is a challenging problem when MT are out-of-network coverage.

2) Outdoor Positioning: Similarly to indoor environment, D2D communications can enable performing cooperative localization in cellular networks, where the BS MT links are supplemented with the inter-MT links that provide relative location information between the MTs [5].

In [39], authors investigate D2D communication capabilities to empower cooperative positioning in $5 \mathrm{G}$ for high-density scenarios, which have the potential to enable centimeter-level accuracy positioning estimates. More specifically, MT receive and exploit signals from other MT in proximity. The pseudo range estimates (e.g., TDOA) between MTs is then used to increase the positioning accuracy. Authors demonstrate that at densities greater than 1,100 MT per square kilometer, submeter positioning accuracy can be provided with the outage probability converging to zero.

In [49], a cooperative localization technique using RF Pattern Matching for LTE systems is proposed, wherein, by leveraging the $\mathrm{D} 2 \mathrm{D}$ communication protocol, the MTs communicate with both the BS to capture a series of RF measurements and other MT. Simulation results of the proposed cooperative algorithm testify to a significant improvement of the positioning accuracy in cellular LTE networks.

In [50], authors focus on the integrated methodology of GNSS and D2D measurements in 5G communication system to achieve a high-level accuracy. In addition, authors state that the high-dense property of $5 \mathrm{G}$ networks also eases the process of obtaining sufficient D2D measurements to achieve any-time ubiquitous positioning as $\mathrm{D2D}$ benefits from the high density of connected MT The positioning accuracy of GNSS system is improved up to $56.2 \%$ compared to non-cooperative approach.

The collaboration among the communication, signal processing, and control sub-systems in ITS systems is considered in [48], while [14], with reference to [TS, discusses the key characteristics of $5 \mathrm{G}$ mmWave positioning for vehicular networking that can benefit from $5 \mathrm{G}$ technologies, such as D2D Moreover, even in the absence of reference stations, D2D cooperative localization allows for relative positioning.

In [51], authors investigate content distribution problems in D2D-based cooperative vehicular networks. The same authors propose the algorithm to achieve dependable content distribution through highly dynamic and unreliable D2D. Vehicle-to-Vehicle (V2V) links by combining big data-based vehicle trajectory prediction with coalition formation gamebased resource allocation.

By studying Table III one can learn that D2D-aided cooperative positioning provides the increased accuracy compared to non-cooperative methods. It is important to emphasize that D2D also helps extend the coverage since it allows for relative position estimation, even in the absence of reference stations. Further, according to Table $\mathrm{III} 5 \mathrm{5G}$ mobile networks have enhanced positioning capabilities in comparison with previous generations and, with the help of $D 2 D$-aided cellular positioning, can ensure sub-meter accuracy needed by $5 G$ applications and have the potential to enable centimeter-level positioning accuracy.

We note that $D 2 D$ provides two advantages for the positioning accuracy improvement. First, the direct exchange of necessary data between MT can be performed. In this case, both common physical layer estimates (to speed up the local decisions), as well as position information, are exchanged over D2D links to enhance the accuracy of the localization system. Such information, including uncertainties of estimates, is relevant to improve the convergence time of estimation processes in the MT Second, with the implementation of D2D communication capabilities, MTs are inherently receiving signals from each other. These numerous links contain the additional signal observations and, hence, can be used to determine the pseudo-range estimates between MT.

Analyzing Table III one can deduce that the cooperative positioning approach, where additional pseudo-range observations from D2D links are available, is more commonly used by the research community. With this principle, it is possible to estimate distances between MT in the form of pseudo ranges that come on top of MTs-BSs ranging and are used in today's mobile communications systems. Thereby, seamless positioning at a sufficient accuracy level can be achieved. In the case of location information exchange, benefits are in terms of improvement in the location process convergence time. In any case, cooperative D2D-aided positioning in 5G requires the exchange of data between the MT or between MT and the network, which can be achieved by an appropriate position information exchange protocol.

\section{LESSONS LEARNED}

This section summarizes the main lessons learned while exploring the existing research on D2D-based cooperative positioning. In summary, we have learned that:

1. Ultra-dense network deployments may pave the way to provide accurate collaborative positioning. D2D communication between mobile devices together with a high density of small cells in $5 \mathrm{G} N \mathrm{NR}$ networks paves the way for cooperative positioning applications. Moreover, sophisticated resource allocation between users can improve the localization performance, as in [52]. Thus, cooperative D2D-aided positioning in ultra-dense $5 \mathrm{G}$ networks is foreseen to provide continuous high-level accuracy positioning estimations.

2. There is a lack of efficient protocols for cooperative positioning, which needs to be improved. Compared to previous generations of mobile networks, in the upcoming commercial $5 \mathrm{G}$ deployments, significantly higher positioning accuracy is anticipated. This is especially due to, among others, the capability of D2D communication to enable cooperative positioning. Cooperative positioning protocols could exploit the high density of asynchronous and synchronous nodes in future heterogeneous networks independently of the location method. Although preliminary studies have been launched within D2D communications, the exchange of location information among mobile devices, small cells, and BS; needs to be improved to enable the deployment of cooperative 
positioning techniques within the whole network. Thus, the standard should contain a cooperative protocol within the network elements to improve the location information sharing for both communication and positioning purposes. Moreover, recent results in this research direction have already demonstrated the potential for centimeter-level localization accuracy.

3. Cooperative positioning may become complex as the number of collaborators/actors increases. While the positioning accuracy becomes higher when the number of collaborators grows, the complexity also increases. For example, in fully connected mesh network, the number of links established among MTs is $N_{\mathrm{mt}}\left(N_{\mathrm{mt}}-1\right)$ that grows quadratically with number of MT $N_{\mathrm{mt}}$. Therefore, the performance-complexity trade-off is a key issue that still has to be investigated by the research community. Moreover, when the number of possible collaborators exceeds the number of needed collaborators to perform the accurate positioning, the procedure for collaborators selection has to be established. For example, random selection can be determined.

4. There is a trade-off between energy consumption and high transmission rates/low latency. From an energy consumption perspective, minimizing the active interfaces and sending data at best possible data rate will bring the lowest energy usage on the MT side. Thus, for D2D transmission, MTs should keep only the cellular interface active to save energy unless there is a need for very high data rate transmissions, which depends on the application. However, high transmission rates guarantee low latency services. Therefore, finding an optimal power consumption vs. latency trade-off presents itself as an important research direction, especially for high-accuracy positioning. In addition, different from previous cellular technology generations, $5 \mathrm{G} \mathrm{NR}$ is able to meet the future networks' positioning requirements of less than onemeter accuracy.

5. D2D technology reduces location estimation error under NLOS environments. The localization accuracy sharply declines once the receiver enters NLOS environment. Cooperative localization also combats this problem, increases the accuracy, and extends the coverage compared to noncooperative localization. Moreover, it can provide resilient relative localization estimates even when the network infrastructure is not available.

\section{ThE WAY FORWARD: OPEN ISSUES}

In this section, we discuss further aspects related to D2D. based cooperative positioning that still need future investigations by the research and industry communities.

Green Communications. There is a rising interest in developing more energy-efficient protocols and algorithms for a D2D-based collaborative positioning, which can reduce the economic and environmental impact and promote Green Communications. Since, in 5G communication systems, the use of energy and spectrum resources plays a significant role, green communications represent a very timely topic. Also, adaptive power control should be analyzed in order to gain a more detailed understanding, e.g., on the effect of transmissions.

Mobility. In many cases, due to the portability of the nodes, mobility becomes an issue to be considered in the design of these communication protocols. Also, mobility makes localization techniques increasingly less accurate, and these errors usually increase with node speed. Moreover, the impact that anchors' and agents' mobility, needing frequent handovers in a Multi-Radio Access Technology (RAT network, may have on energy consumption has still received limited attention from the research community. On the other side, some studies prove that the positioning accuracy could be improved by leveraging the mobility capabilities of the nodes depending on the level of mobility of the nodes within the network.

Utilization of the Beam Training Period for Positioning. Since highly directional transmissions (e.g., by using mmWave and terahertz bands) are considered as one of the main components of $5 \mathrm{G}$ and $\mathrm{B} 5 \mathrm{G}$ systems, the feasibility of utilizing the beam training period for positioning is of extreme interest.

Synchronization. The network synchronization is a critical aspect of the cellular ranging-based location methods, such as OTDOA, UTDOA, which should not exceed the order of nanoseconds for accurate positioning. Thus, to support ranging-based methods, cellular standards should take into account tight network synchronization requirements. Indoors, e.g., the use of advanced network time protocols or accurate round-trip time should be considered. Moreover, the chosen waveform will impact the synchronization requirements, potentially gaining localization accuracy.

LTE sidelink communication can handle the synchronization problem between MT in distributed cooperative localization systems through the primary sidelink synchronization signal. However, it can be managed only for those MT that are under the eNodeBs coverage, whereas synchronization is a challenging problem when MT are out-of-network coverage.

Higher carrier frequencies increase the resolution of multipaths. Higher carrier frequencies, such as mmWave spectrum, increases the LoS reception probability since any NLoS condition is likely to be blocked due to the severe penetration and propagation properties. Consequently, the channel becomes more sparse in the sense of few dominant multipath components and very few reflected paths. A sparse channel means that it is easier to identify individual specular multipath components that can be used for high-accurate positioning by reducing the risk of positioning errors due to the NLoS bias.

\section{CONCLUSION}

In this work, we provided a survey on the state-of-theart of D2D-aided cooperative positioning, which can be determined as the information exchange among MT intending to increase their localization accuracy. In summary, 5G system positioning capabilities have been enhanced compared to previous generations of communication networks, especially thanks to the D2D technology allowing to perform cooperative positioning in cellular systems. It has already been demonstrated that in such systems, the positioning accuracy of $1 \mathrm{~m}$ and below can be reached, thereby satisfying $5 \mathrm{G}$ application requirements. More importantly, it has the visible potential to offer centimeter-level accuracy. Nevertheless, there still remain plenty of challenges to be solved by the research community. Among them, the need for efficient protocols, positioning 
complexity, and green communication issues, among others, have to be addressed.

\section{APPENDIX I \\ LIST OF ACRONYMS}

$\begin{array}{ll}\text { 2G } & \text { second-generation mobile telecommunications } \\ \text { 3G } & \text { third-generation mobile telecommunications } \\ \text { 3GPP } & \text { the 3rd Generation Partnership Project } \\ \text { 4G } & \text { fourth-generation mobile telecommunications } \\ \text { 5G } & \text { fifth-generation } \\ \text { AOA } & \text { Angle Of Arrival } \\ \text { AOD } & \text { Angle Of Departure } \\ \text { AP } & \text { Access Point } \\ \text { B5G } & \text { beyond 5G } \\ \text { BLE } & \text { Bluetooth Low Energy } \\ \text { BS } & \text { Base Station } \\ \text { CAM } & \text { Cooperative Awareness Message } \\ \text { D2D } & \text { Device-to-Device } \\ \text { DENM } & \text { Decentralized Environmental Notification Message } \\ \text { DL } & \text { Downlink } \\ \text { GIS } & \text { Geographic Information System } \\ \text { GNSS } & \text { Global Navigation Satellite System } \\ \text { GPS } & \text { Global Positioning System } \\ \text { GSM } & \text { Global System for Mobile Communications } \\ \text { IEEE } & \text { Institute of Electrical and Electronics Engineers } \\ \text { IIPS } & \text { Infrastructure-based Indoor Positioning System } \\ \text { IoT } & \text { Internet of Things } \\ \text { IR } & \text { Identifiable Infrared } \\ \text { ISM } & \text { Industrial, Scientific and Medical } \\ \text { ITS } & \text { Intelligent Transportation System } \\ \text { KPI } & \text { Key Performance Indicators } \\ \text { LBS } & \text { Location-based Services } \\ \text { LoS } & \text { Line-of-Sight } \\ \text { LTE } & \text { Long Term Evolution } \\ \text { MIMO } & \text { Multiple-Input-Multiple-Output } \\ \text { MKF } & \text { Multi-Kalman Filter } \\ \text { mmWave } & \text { Millimeter Wave } \\ \text { MT } & \text { Mobile Terminal } \\ \text { NLOS } & \text { Non-Line-of-Sight } \\ \text { NR } & \text { New Radio } \\ \text { OTDOA/UTDOA Observed/Uplink Time Difference of Arrival } \\ \text { PPP } & \text { Precise Point Positioning } \\ \text { QoS } & \text { Quality of Service } \\ \text { RAT } & \text { Radio Access Technology } \\ \text { RF } & \text { Radio Frequency } \\ \text { RFID } & \text { Radio Frequency Identification } \\ \text { RSSI } & \text { Received Signal Strength } \\ \text { RTK } & \text { Real-Time Kinematic } \\ \text { SNR } & \text { Signal-to-Noise Ratio } \\ \text { TBS } & \text { Terrestrial Beacon Systems } \\ \text { TDOA } & \text { Time Difference Of Arrival } \\ \text { TOA } & \text { Time Of Arrival } \\ \text { TTFF } & \text { Time to First Fix } \\ \text { UAV } & \text { Unmanned Aerial Vehicle } \\ \text { UE } & \text { User Equipment } \\ \text { UL } & \text { Uplink } \\ \text { UWB } & \text { Ultra-Wide Band } \\ \text { V2V } & \text { Vehicle-to-Vehicle } \\ \text { V2X } & \text { Vehicle-to-Everything } \\ \text { Wi-Fi } & \text { Wireless Fidelity } \\ \text { WLAN } & \text { Wireless Local Area Network } \\ \text { WPAN } & \text { Wireless Personal Area Network } \\ \text { WSN } & \text { Wireless Sensor Network } \\ & \end{array}$

\section{REFERENCES}

[1] R. Hattachi and J. Erfanian, "5G White Paper," Next Generation Mobile Networks Alliance, 2015.

[2] 3GPP, "Feasibility Study on New Services and Markets Technology Enablers for Critical Communications," 3GPP TR 22.862 V14.1.0, Sep. 2016.

[3] J. Talvitie, T. Levanen, M. Koivisto, et al., "Positioning of High-Speed Trains Using 5G New Radio Synchronization Signals," in 2018 IEEE Wireless Communications and Networking Conference (WCNC), IEEE, 2018, pp. 1-6.
[4] J. Talvitie, T. Levanen, M. Koivisto, et al., "Positioning and LocationAware Communications for Modern Railways with 5G New Radio," IEEE Communications Magazine, vol. 57, no. 9, pp. 24-30, 2019.

[5] P. Zhang, J. Lu, Y. Wang, et al., "Cooperative Localization in 5G Networks: A Survey," Ict Express, vol. 3, no. 1, pp. 27-32, 2017.

[6] R. M. Buehrer, H. Wymeersch, and R. M. Vaghefi, "Collaborative Sensor Network Localization: Algorithms and Practical Issues," Proceedings of the IEEE, vol. 106, no. 6, pp. 1089-1114, 2018.

[7] S. Khandker, J. Torres-Sospedra, and T. Ristaniemi, "Improving RF Fingerprinting Methods by Means of D2D Communication Protocol," Electronics, vol. 8, no. 1, p. 97, 2019.

[8] 3GPP, "Study on Positioning Use Cases (Release 16)," 3GPP TR 22.872 V16.1.0, Sep. 2018.

[9] J. A. del Peral-Rosado, R. Raulefs, J. A. López-Salcedo, et al., "Survey of Cellular Mobile Radio Localization Methods: From 1G to 5G," IEEE Communications Surveys \& Tutorials, vol. 20, no. 2, pp. 1124 $1148,2017$.

[10] 3GPP, "Service Requirements for the 5G System (Release 18)," 3GPP TS 22.261 V18.2.0, Tech. Rep., Mar. 2021.

[11] _ "Service Requirements for Cyber-Physical Control Applications in Vertical Domains (Release 18)," 3GPP TS 22.104 V18.0.0, Tech. Rep., Mar. 2021.

[12] S. Bartoletti, A. Conti, D. Dardari, et al., "5G Localization and Context-Awareness," University of Bologna, University of Ferrara, 2018.

[13] 3GPP, "Study on Indoor Positioning Enhancements for UTRA and LTE (Release 13)," 3GPP TR 37.857 V13.1.0, Dec. 2015.

[14] H. Wymeersch, G. Seco-Granados, G. Destino, et al., "5G mmWave Positioning for Vehicular Networks," IEEE Wireless Communications, vol. 24, no. 6, pp. 80-86, 2017.

[15] H. Wymeersch, N. Garcia, H. Kim, et al., "5G mmWave Downlink Vehicular Positioning," in 2018 IEEE Global Communications Conference (GLOBECOM), IEEE, 2018, pp. 206-212.

[16] C. Ma, J. Yang, J. Chen, et al., "Indoor and Outdoor Positioning System based on Navigation Signal Simulator and Pseudolites," Advances in Space Research, vol. 62, no. 9, pp. 2509-2517, 2018.

[17] P. Pascacio, S. Casteleyn, J. Torres-Sospedra, et al., "Collaborative Indoor Positioning Systems: A Systematic Review," Sensors, vol. 21, no. 3, p. 1002, 2021.

[18] J. Palacios, P. Casari, and J. Widmer, "JADE: Zero-Knowledge Device Localization and Environment Mapping for Millimeter Wave Systems," in IEEE INFOCOM 2017-IEEE Conference on Computer Communications, IEEE, 2017, pp. 1-9.

[19] O. Kanhere and T. S. Rappaport, "Position Locationing for Millimeter Wave Systems," in 2018 IEEE Global Communications Conference (GLOBECOM), IEEE, 2018, pp. 206-212.

[20] N. Garcia, H. Wymeersch, E. G. Larsson, et al., "Direct Localization for Massive MIMO," IEEE Transactions on Signal Processing, vol. 65 , no. 10 , pp. 2475-2487, 2017.

[21] H. Wymeersch, J. Lien, and M. Z. Win, "Cooperative Localization in Wireless Networks," Proceedings of the IEEE, vol. 97, no. 2, pp. 427450, 2009.

[22] N. Garcia, A. M. Haimovich, J. A. Dabin, et al., "Direct Localization of Emitters Using Widely Spaced Sensors in Multipath Environments," in 2014 48th Asilomar Conference on Signals, Systems and Computers, IEEE, 2014, pp. 695-700.

[23] N. Pirzada, M. Y. Nayan, F. Subhan, et al., "Comparative Analysis of Active and Passive Indoor Localization Systems," AASRI Procedia, vol. 5, pp. 92-97, 2013.

[24] Z. Wu, Q. Xu, J. Li, et al., "Passive Indoor Localization based on SCI and Naive Bayes Classification," IEEE Transactions on Systems, Man, and Cybernetics: Systems, vol. 48, no. 9, pp. 1566-1577, 2017.

[25] A. Das and G. Dubbelman, "An Experimental Study on Relative and Absolute Pose Graph Fusion for Vehicle Localization," in 2018 IEEE Intelligent Vehicles Symposium (IV), IEEE, 2018, pp. 630-635.

[26] P. Zhang and Q. Wang, "On Using the Relative Configuration to Explore Cooperative Localization," IEEE Transactions on Signal Processing, vol. 62, no. 4, pp. 968-980, 2014.

[27] R. Klus, L. Klus, D. Solomitckii, et al., "Deep Learning Based Localization and HO Optimization in 5G NR Networks," in 2020 International Conference on Localization and GNSS (ICL-GNSS), IEEE, 2020, pp. 1-6.

[28] A. Ometov, A. Orsino, L. Militano, et al., "A Novel Security-Centric Framework for D2D Connectivity based on Spatial and Social Proximity," Computer Networks, vol. 107, pp. 327-338, 2016. 
[29] H. Sun, Z. Zhang, R. Q. Hu, et al., "Wearable Communications in 5G: Challenges and Enabling Technologies," ieee vehicular technology magazine, vol. 13, no. 3, pp. 100-109, 2018.

[30] A. Ometov, V. Shubina, L. Klus, et al., "A Survey on Wearable Technology: History, State-of-the-Art and Current Challenges," Computer Networks, vol. 193, p. 108 074, 2021.

[31] A. Asadi and V. Mancuso, "WiFi Direct and LTE D2D in action," in 2013 IFIP Wireless Days (WD), IEEE, 2013, pp. 1-8.

[32] M. Höyhtyä, O. Apilo, and M. Lasanen, "Review of Latest Advances in 3GPP Standardization: D2D Communication in 5G Systems and its Energy Consumption Models," Future Internet, vol. 10, no. 1,3, 2018.

[33] S. Saafi, J. Hosek, and A. Kolackova, "Cellular-enabled Wearables in Public Safety Networks: State of the Art and Performance Evaluation," in 2020 12th International Congress on Ultra Modern Telecommunications and Control Systems and Workshops (ICUMT), IEEE, 2020, pp. 201-207.

[34] M. M. Alam, D. B. Arbia, and E. B. Hamida, "Research Trends in Multi-Standard Device-to-Device Communication in Wearable Wireless Networks," in International Conference on Cognitive Radio Oriented Wireless Networks, Springer, 2015, pp. 735-746.

[35] S.-Y. Lien, D.-J. Deng, C.-C. Lin, et al., "3GPP NR Sidelink Transmissions Toward 5G V2X," IEEE Access, vol. 8, pp. 35 368-35 382, 2020.

[36] X. Wang and S. Akoum, Forward Compatible New Radio Sidelink Slot Format Signalling, US Patent 10,873,944, Dec. 2020.

[37] H. Bagheri, Z. Liu, H. Lee, et al., "5G NR-V2X: Towards Connected and Cooperative Autonomous Driving," arXiv preprint arXiv:2009.03638, 2020.

[38] M. Koivisto, M. Costa, J. Werner, et al., "Joint Device Positioning and Clock Synchronization in 5G Ultra-Dense Networks," IEEE Transactions on Wireless Communications, vol. 16, no. 5, pp. 2866-2881, 2017.

[39] A. Dammann, R. Raulefs, and S. Zhang, "On Prospects of Positioning in 5G," in 2015 IEEE International Conference on Communication Workshop (ICCW), IEEE, 2015, pp. 1207-1213.

[40] X. Cui, T. A. Gulliver, H. Song, et al., "Real-Time Positioning based on Millimeter Wave Device to Device Communications," IEEE Access, vol. 4, pp. 5520-5530, 2016.

[41] X. Cui, T. A. Gulliver, J. Li, et al., "Vehicle Positioning Using 5G millimeter-wave Systems," IEEE Access, vol. 4, pp. 6964-6973, 2016.

[42] A. Papapostolou, W. Xiao, and H. Chaouchi, "Cooperative Fingerprintbased Indoor Localization Using Self-Organizing Maps," in 2011 7th International Wireless Communications and Mobile Computing Conference, IEEE, 2011, pp. 1814-1819.

[43] T. Iwase and R. Shibasaki, "Infra-free Indoor Positioning Using Only Smartphone Sensors," in International Conference on Indoor Positioning and Indoor Navigation, IEEE, 2013, pp. 1-8.

[44] P. Raveneau, S. D'Alu, and H. Rivano, "Localisation based on WiFi Fingerprints: A Crowdsensing Approach with a Device-to-Device Aim," in 2017 IEEE International Conference on Pervasive Computing and Communications Workshops (PerCom Workshops), IEEE, 2017, pp. 321-325.

[45] M. Karlsson and F. Karlsson, "Cooperative Indoor Positioning by Exchange of Bluetooth Signals and State Sstimates Between Users," in 2016 European Control Conference (ECC), IEEE, 2016, pp. 14401444.

[46] R. M. Vaghefi and R. M. Buehrer, "Improving Positioning in LTE through Collaboration," in 2014 11th Workshop on Positioning, Navigation and Communication (WPNC), IEEE, 2014, pp. 1-6.

[47] 3GPP, "Study on Indoor Positioning Enhancements for UTRA and LTE (Release 13)," 3GPP TR 37.857 V13.1.0, Jan. 2016.

[48] H. Wymeersch, G. R. de Campos, P. Falcone, et al., "Challenges for Cooperative ITS: Improving Road Safety Through the Integration of Wireless Communications, Control, and Positioning," in 2015 International Conference on Computing, Networking and Communications (ICNC), IEEE, 2015, pp. 573-578.

[49] R. M. Vaghefi and R. M. Buehrer, "Cooperative RF Pattern Matching Positioning for LTE Cellular Systems," in 2014 IEEE 25th Annual International Symposium on Personal, Indoor, and Mobile Radio Communication (PIMRC), IEEE, 2014, pp. 264-269.

[50] L. Yin, Q. Ni, and Z. Deng, "A GNSS/5G Integrated Positioning Methodology in D2D Communication Networks," IEEE Journal on Selected Areas in Communications, vol. 36, no. 2, pp. 351-362, 2018.

[51] Z. Zhou, H. Yu, C. Xu, et al., "Dependable Content Distribution in D2D-based Cooperative Vehicular Networks: A Big Data-Integrated Coalition Game Approach," IEEE Transactions on Intelligent Transportation Systems, vol. 19, no. 3, pp. 953-964, 2018.
[52] R. Raulefs, S. Zhang, and C. Mensing, "Bound-based Spectrum Allocation for Cooperative Positioning," Transactions on Emerging Telecommunications Technologies, vol. 24, no. 1, pp. 69-83, 2013.

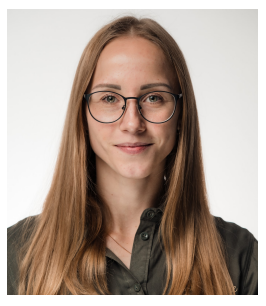

Nadezhda Chukhno is an Early Stage Researcher at A-WEAR and Doctoral Researcher at University Mediterranea of Reggio Calabria, Italy and Jaume I University, Spain. She graduated from RUDN University, Russia, and received her B.Sc. in Business Informatics (2017) and M.Sc. in Fundamental Informatics and Information technologies (2019). Her current research activity mainly focuses on wireless communications, D2D, and wearable technologies.

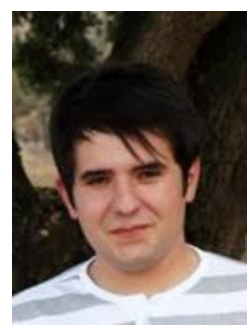

Sergio Trilles has a $\mathrm{PhD}$ in Integration of Geospatial Information from the Jaume I University in 2015 and he is currently a post-doctoral fellow at University Jaime I, holding a Juan de la Cierva-Incorporacion fellowship. His research lines are centre on geospatial fields such as the Internet of Things (sensors), interoperability, geoprocessing or web mapping. He is author of more than fifty journal and conference peerreviewed publications.

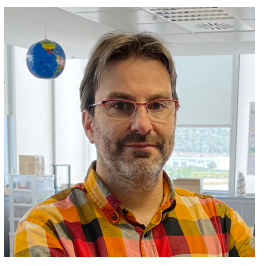

Joaquín Torres-Sospedra is CEO at UBIK Geospatial Solutions. He has a PhD since 2011 about Ensembles of Neural Networks and $\mathrm{Ma}$ chine Learning from Universitat Jaume I. He has authored more than 120 articles in journals and conference proceedings. His current research interests include indoor positioning solutions based on Wi-Fi \& BLE, Machine Learning and Evaluation. He is the chair of the Smartphonebased track of IPIN Competition and the IPIN International Standards Committee.

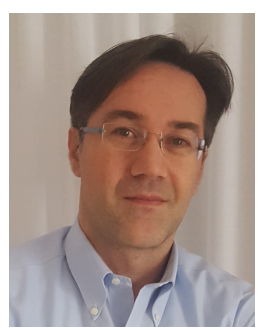

Antonio lera graduated in computer engineering from the University of Calabria in 1991, and received a Master's degree in IT from CEFRIEL/Politecnico di Milano in 1992 and a Ph.D. degree from the University of Calabria in 1996. From 1997 to 2019 he has been with the University Mediterranea, Italy, and currently holds the position of full professor of Telecommunications at the University of Calabria, Italy. His research interests include next generation mobile and wireless systems, and the Internet of Things.

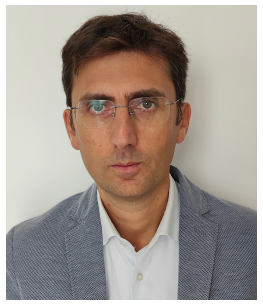

Giuseppe Araniti (Senior Member, IEEE) received the Laurea degree and the Ph.D. degree in electronic engineering from the University Mediterranea of Reggio Calabria, Italy, in 2000 and 2004, respectively. He is currently an Assistant Professor of telecommunications with the University Mediterranea of Reggio Calabria. His major area of research is on $5 \mathrm{G} / 6 \mathrm{G}$ networks and it includes personal communications, enhanced wireless and satellite systems, traffic and radio resource management, multicast and broadcast services, device-to-device (D2D), and machine-type communications (M2M/MTC). 